\title{
THE MAPPINGS OF DEGREE 1
}

\author{
MARIA N. KREIN
}

Received 26 June 2005; Accepted 1 July 2005

The maps of the form $f(x)=\sum_{i=1}^{n} a_{i} \cdot x \cdot b_{i}$, called 1-degree maps, are introduced and investigated. For noncommutative algebras and modules over them 1-degree maps give an analogy of linear maps and differentials. Under some conditions on the algebra $\mathscr{A}$, contractibility of the group of 1-degree isomorphisms is proved for the module $l_{2}(\mathscr{A})$. It is shown that these conditions are fulfilled for the algebra of linear maps of a finitedimensional linear space. The notion of 1-degree map gives a possibility to define a nonlinear Fredholm map of $l_{2}(\mathscr{A})$ and a Fredholm manifold modelled by $l_{2}(\mathscr{A})$. 1-degree maps are also applied to some problems of Markov chains.

Copyright (C) 2006 Maria N. Krein. This is an open access article distributed under the Creative Commons Attribution License, which permits unrestricted use, distribution, and reproduction in any medium, provided the original work is properly cited.

\section{Motivation}

For the last several decades various algebras and algebra modules have been intensively investigated. Module maps are traditionally called linear if they preserve addition and multiplication by elements of the algebra (for noncommutative algebra-left or right multiplication). For nonlinear maps of modules it is easy to give a definition of the derivative similar to Freche derivative in linear spaces. In the case of noncommutative algebra, even simplest nonlinear maps, for example, power maps of the algebra (i.e., here a "onedimensional" module) do not have such derivatives, however there exists analogue of the differential, containing only first power of the "argument increment." Namely,

$$
\begin{gathered}
(x+\Delta x)^{2}-x^{2}=x \cdot \Delta x+\Delta x \cdot x+(\Delta x)^{2}, \\
(x+\Delta x)^{3}-x^{3}=x^{2} \cdot \Delta x+x \cdot \Delta x \cdot x+\Delta x \cdot x^{2}+(\Delta x)^{2} \cdot x+\Delta x \cdot x \cdot \Delta x+x \cdot(\Delta x)^{2}+(\Delta x)^{3},
\end{gathered}
$$

and so forth. 
2 The mappings of degree 1

This was the reason for the author to introduce and investigate the maps as $f_{1}(\Delta x)=$ $x \cdot \Delta x+\Delta x \cdot x$ and $f_{2}(\Delta x)=x^{2} \cdot \Delta x+x \cdot \Delta x \cdot x+\Delta x \cdot x^{2}$.

\section{1-degree maps of algebras}

Let $\mathscr{A}$ be an algebra.

Definition $2.1[2-10]$. The map $f: \mathscr{A} \rightarrow \mathscr{A}$ is called a mapping of degree 1 (1-degree map for short) if $f(x)=\sum_{i=1}^{n} a_{i} \cdot x \cdot b_{i}$ for some $n \in N$ and $a_{1}, a_{2}, \ldots, a_{n}, b_{1}, b_{2}, \ldots, b_{n} \in \mathscr{A}$.

By $d_{1}(\mathscr{A})$ denote the set of 1 -degree maps.

Obviously, for a commutative algebra $\mathscr{A}$ a 1-degree map is a trivial multiplication by a specified element. That is why $\mathscr{A}$ will denote further a noncommutative algebra.

Let $\operatorname{Map}(\mathscr{A})$ be the algebra of all maps from $\mathscr{A}$ to $\mathscr{A}$ with usual addition and multiplication by number and with map composition as element's multiplication.

Let $A$ be $A$ without the element's multiplication. $A$ is a linear space over some number field $F$. Let $L(A)$ denote the algebra of its linear operators. It is obvious that $d_{1}(\mathscr{A}) \subset$ $L(A) \subset \operatorname{Map}(\mathscr{A})$.

Definition 2.2. We say that $\mathscr{A}$ is 1 -algebra if $d_{1}(\mathscr{A})=L(A)$.

Theorem 2.3. If $\mathscr{A}=L\left(F^{n}\right)$ for some field $F$, then $\mathscr{A}$ is a 1-algebra.

Proof. It is sufficient to prove that $L(A) \subset d_{1}(\mathscr{A})$. Let $X \in \mathscr{A}$. Since $\mathscr{A}=L\left(F^{n}\right)$, then $\mathscr{A}$ is isomorphic to the algebra of square $F$-matrices, that is, $A$ is $n^{2}$-dimensional linear space with matrices

$$
P_{i j}=\left(\begin{array}{ccccc}
0 & \cdots & 0 & \cdots & 0 \\
\vdots & & \vdots & & \vdots \\
0 & \cdots & 1 & \cdots & 0 \\
\vdots & & \vdots & & \vdots \\
0 & \cdots & 0 & \cdots & 0
\end{array}\right)
$$

as a basis. (Matrix $P_{i j}$ consists of zeroes except 1 at the intersection of $i$ th row and $j$ th column.) If

$$
X=\left(\begin{array}{ccc}
x_{11} & \cdots & x_{1 n} \\
\vdots & & \vdots \\
x_{n 1} & \cdots & x_{n n}
\end{array}\right)
$$

$X=\sum_{i=1}^{n} \sum_{j=1}^{n} x_{i j} P_{i j}$ and $f(X)=\sum_{i=1}^{n} \sum_{j=1}^{n} x_{i j} f\left(P_{i j}\right)$ for $f \in L(A)$. If $x_{i j} \neq 0$, then $P_{i j}=$ $\left(1 / x_{i j}\right) P_{i i} X P_{j j}=\left(\left(1 / x_{i j}\right) P_{i i}\right) X P_{j j}=P_{i i} X\left(\left(1 / x_{i j}\right) P_{j j}\right)$. $\left(P_{i j}\right.$ can be obtained from $X$ in another way as well.) 
Let

$$
f\left(P_{i j}\right)=\left(\begin{array}{ccc}
a_{11}^{i j} & \cdots & a_{1 n}^{i j} \\
\vdots & & \vdots \\
a_{n 1}^{i j} & \cdots & a_{n n}^{i j}
\end{array}\right)=\sum_{k=1}^{n} \sum_{l=1}^{n} a_{k l}^{i j} P_{k l} .
$$

For any $i, j \in\{1,2, \ldots, n\}$ we have $P_{k l}=P_{k i} P_{i j} P_{j l}$. Hence

$$
\begin{aligned}
f(X) & =\sum_{i=1}^{n} \sum_{j=1}^{n} x_{i j} \sum_{k=1}^{n} \sum_{l=1}^{n} a_{k l}^{i j} P_{k i} P_{i j} P_{j l} \\
& =\sum_{x_{i j} \neq 0} \sum_{k=1}^{n} \sum_{l=1}^{n} a_{k l}^{i j} P_{k i} x_{i j} \frac{1}{x_{i j}} P_{i i} X P_{j j} P_{j l} \\
& =\sum_{x_{i j} \neq 0} \sum_{k=1}^{n} \sum_{l=1}^{n} a_{k l}^{i j} P_{k i} P_{i i} X P_{j j} P_{j l} .
\end{aligned}
$$

If $x_{i j}=0$, then $P_{i i} X P_{j j}$ is a nil-matrix. So,

$$
f(X)=\sum_{i=1}^{n} \sum_{j=1}^{n} \sum_{k=1}^{n} \sum_{l=1}^{n}\left(a_{k l}^{i j} P_{k i} P_{i i}\right) X\left(P_{j j} P_{j l}\right)=\sum_{i=1}^{n} \sum_{j=1}^{n} \sum_{k=1}^{n} \sum_{l=1}^{n}\left(a_{k l}^{i j} P_{k i}\right) X\left(P_{j l}\right),
$$

that is, $f \in d_{1}(\mathscr{A})$. Therefore $d_{1}(\mathscr{A})=L(A)$. The theorem follows.

Replace the multiplication $x y$ in $\mathscr{A}$ by $x \odot y=y x$ and denote the new algebra by $\widetilde{\mathscr{A}}$. There is an algebra homomorphism (epimorphism) $h: \mathscr{A} \otimes \widetilde{\mathscr{A}} \rightarrow d_{1}(\mathscr{A}), h\left(\sum_{i=1}^{n} a_{i} \otimes\right.$ $\left.b_{i}\right)=f$, where $f(x)=\sum_{i=1}^{n} a_{i} \cdot x \cdot b_{i}$. Ker $h$ is not trivial. Some conditions, under which $\sum_{i=1}^{n} a_{i} \otimes b_{i} \in \operatorname{Ker} h$, are stated below.

THEOREM 2.4. If $\mathscr{A}=L(E)$ for some linear space $E$ over any field $F$, then

(1) $a \otimes b \in$ Ker $h$ if and only if $a=0$ or $b=0$;

(2) for $a, b, c, d \neq 0$ one gets $a \otimes b-c \otimes d \in \operatorname{Ker} h$ if and only if $\exists \lambda \in F$ is such that $a=\lambda c$ and $b=(1 / \lambda) d$.

Proof. (1) If $a=0$ or $b=0$, then, obviously, for any $x \in \mathscr{A}$ one gets $a x b=0$, that is, $a \otimes b \in \operatorname{Ker} h$. If $a \neq 0$ and $b \neq 0$, then there exist $e_{1}, e_{2} \in E$ such that $b\left(e_{1}\right) \neq 0$ and $a\left(e_{2}\right) \neq$ 0 . Since $\mathscr{A}$ is the algebra of all linear maps from $E$ to $E$, there is $x \in \mathscr{A}$ such that $x\left(b\left(e_{1}\right)\right)=$ $e_{2}$. So, $(a x b)\left(e_{1}\right)=(a x)\left(b\left(e_{1}\right)\right)=a\left(x\left(b\left(e_{1}\right)\right)\right)=a\left(e_{2}\right) \neq 0$. Therefore, $a x b \neq 0 \in \mathscr{A}$, thus $a \otimes b \notin \operatorname{Ker} h$.

(2) Suppose that $a, b, c, d \neq 0$ but there is no $\lambda \in F$ with properties formulated in this theorem. (If such $\lambda$ exists, then, evidently, $a \otimes b-c \otimes d \in$ Ker $h$.) Consider the following cases.

(2.1) There exists $\alpha \in F$ such that $\alpha \neq 0, a=\alpha c, b \neq(1 / \alpha) d$. As $b \neq(1 / \alpha) d, b, d \neq 0$, we can find $e_{1} \in E$ such that $e_{1} \neq 0, b\left(e_{1}\right) \neq(1 / \alpha) d\left(e_{1}\right)$, and at least one element $b\left(e_{1}\right)$ or $d\left(e_{1}\right)$ is not equal to zero. As $a, c \neq 0, a=\alpha c$, we can find $e_{2} \in E$ such that $e_{2} \neq 0$, $a\left(e_{2}\right)=\alpha c\left(e_{2}\right) \neq 0$. 
If $b\left(e_{1}\right)=0$, then for any $x \in \mathscr{A}(a x b)\left(e_{1}\right)=0$. But again as far as $\mathscr{A}=L(E)$ is the algebra of all linear maps from $E$ to $E$, there exists $x \in \mathscr{A}$ such that $x\left(d\left(e_{1}\right)\right)=e_{2}$ and $(c x d)\left(e_{1}\right)=c\left(x\left(d\left(e_{1}\right)\right)\right)=c\left(e_{2}\right) \neq 0$. So, $(a x b)\left(e_{1}\right) \neq(c x d)\left(e_{1}\right)$ and $a x b \neq c x d$, that is, $a \otimes$ $b-c \otimes d \notin \operatorname{Ker} h$.

If $d\left(e_{1}\right)=0$, then for any $x \in \mathscr{A}(c x d)\left(e_{1}\right)=0$ and we take $x \in \mathscr{A}$ such that $x\left(b\left(e_{1}\right)\right)=$ $e_{2}$. We obtain $(a x b)\left(e_{1}\right)=a\left(x\left(b\left(e_{1}\right)\right)\right)=a\left(e_{2}\right) \neq 0$, that is, $(a x b)\left(e_{1}\right) \neq(c x d)\left(e_{1}\right), a x b \neq$ $c x d, a \otimes b-c \otimes d \notin$ Kerh.

If $b\left(e_{1}\right) \neq 0, d\left(e_{1}\right) \neq 0$, we take $x \in \mathscr{A}$ such that $x\left(b\left(e_{1}\right)\right)=e_{2}, x\left(d\left(e_{1}\right)\right)=\beta e_{2}$, where $\beta \neq 0, \alpha \neq \beta$. Therefore $(a x b)\left(e_{1}\right)=a\left(x\left(b\left(e_{1}\right)\right)\right)=a\left(e_{2}\right)=\alpha c\left(e_{2}\right)=\alpha c\left((1 / \beta) x\left(d\left(e_{1}\right)\right)\right)=$ $(\alpha / \beta) c\left(x\left(d\left(e_{1}\right)\right)\right)=(\alpha / \beta)(c x d)\left(e_{1}\right) \neq(c x d)\left(e_{1}\right)$ and $a x b \neq c x d$, that is, $a \otimes b-c \otimes d \notin$ Kerh.

(2.2) There exists $\alpha \in F$ such that $\alpha \neq 0, a \neq \alpha c, b=(1 / \alpha) d$. As $b=(1 / \alpha) d \neq 0$, there exists $e_{1} \in E$ such that $e_{1} \neq 0, b\left(e_{1}\right)=(1 / \alpha) d\left(e_{1}\right) \neq 0$. As $a, c \neq 0, a \neq \alpha c$, there exists $e_{2} \in$ $E$ such that $e_{2} \neq 0, a\left(e_{2}\right) \neq \alpha c\left(e_{2}\right)$, and at least one element $a\left(e_{2}\right)$ or $c\left(e_{2}\right)$ is not equal to zero. Let us take $x \in \mathscr{A}$ such that $x\left(b\left(e_{1}\right)\right)=e_{2}$.

If one element, either $a\left(e_{2}\right)$, or $c\left(e_{2}\right)$ is equal to zero, then, obviously, $(a x b)\left(e_{1}\right) \neq$ $(c x d)\left(e_{1}\right)$ because only one of these elements is equal to zero.

If $a\left(e_{2}\right) \neq 0, c\left(e_{2}\right) \neq 0$, then $(a x b)\left(e_{1}\right)=a\left(x\left(b\left(e_{1}\right)\right)\right)=a\left(e_{2}\right) \neq \alpha c\left(e_{2}\right)=\alpha c\left(x\left(b\left(e_{1}\right)\right)\right)=$ $\alpha c\left(x\left((1 / \alpha) d\left(e_{1}\right)\right)\right)=\alpha(1 / \alpha) c\left(x\left(d\left(e_{1}\right)\right)\right)=(c x d)\left(e_{1}\right)$, hence $a x b \neq c x d$. Thus $a \otimes b-c \otimes$ $d \notin \operatorname{Ker} h$.

(2.3) $a \neq \alpha c, b \neq(1 / \alpha) d$ for each $\alpha \in F, \alpha \neq 0$. As $b \neq 0$, there exists $e_{1} \in E$ such that $e_{1} \neq 0, b\left(e_{1}\right) \neq 0$.

If $d\left(e_{1}\right)=0$, then take $e_{2} \in E$ such that $e_{2} \neq 0, a\left(e_{2}\right) \neq 0$ that exists because $a \neq 0$ and $x \in \mathscr{A}$ is such that $x\left(b\left(e_{1}\right)\right)=e_{2}$. Then $(a x b)\left(e_{1}\right)=a\left(e_{2}\right) \neq 0=(b x d)\left(e_{1}\right)$, therefore $a x b \neq$ $c x d$ and $a \otimes b-c \otimes d \notin$ Ker $h$.

If $d\left(e_{1}\right) \neq 0$ and there exists $\gamma \in F$ such that $\gamma \neq 0, b\left(e_{1}\right)=\gamma d\left(e_{1}\right)$, then take $e_{2} \in E$ and $x \in \mathscr{A}$ such that $e_{2} \neq 0, a\left(e_{2}\right) \neq(1 / \gamma) c\left(e_{2}\right), x\left(b\left(e_{1}\right)\right)=e_{2}$. We obtain $(a x b)\left(e_{1}\right)=a\left(e_{2}\right) \neq$ $(1 / \gamma) c\left(e_{2}\right)=(1 / \gamma) c\left(x\left(b\left(e_{1}\right)\right)\right)=(1 / \gamma) c\left(x\left(\gamma d\left(e_{1}\right)\right)\right)=(1 / \gamma) \gamma c\left(x\left(d\left(e_{1}\right)\right)\right)=(c x d)\left(e_{1}\right)$. Thus $a x b \neq c x d$ and $a \otimes b-c \otimes d \notin$ Kerh .

If $b\left(e_{1}\right)$ and $d\left(e_{1}\right)$ are linearly independent (therefore $b\left(e_{1}\right) \neq 0$ and $d\left(e_{1}\right) \neq 0$ ), then take $e_{2} \in E, x \in \mathscr{A}$ such that $e_{2} \neq 0, x\left(b\left(e_{1}\right)\right)=e_{2}$. If $a\left(x\left(b\left(e_{1}\right)\right)\right) \neq c\left(x\left(d\left(e_{1}\right)\right)\right)$, then $a x b \neq$ $c x d$ and $a \otimes b-c \otimes d \notin$ Ker $h$. If it appears to be $a\left(x\left(b\left(e_{1}\right)\right)\right)=c\left(x\left(d\left(e_{1}\right)\right)\right)$, then replace $x$ by $\tilde{x}$ such that $\tilde{x}\left(b\left(e_{1}\right)\right)=x\left(b\left(e_{1}\right)\right), \tilde{x}\left(d\left(e_{1}\right)\right)=\delta x\left(d\left(e_{1}\right)\right)$ where $\delta \neq 0, \delta \neq 1$. Thus we ob$\operatorname{tain}(a \tilde{x} b)\left(e_{1}\right)=a\left(\tilde{x}\left(b\left(e_{1}\right)\right)\right)=a\left(x\left(b\left(e_{1}\right)\right)\right)=c\left(x\left(d\left(e_{1}\right)\right)\right)=c\left((1 / \delta) \tilde{x}\left(d\left(e_{1}\right)\right)\right)=(1 / \delta) c(\tilde{x}(d$ $\left.\left.\left(e_{1}\right)\right)\right) \neq c\left(\tilde{x}\left(d\left(e_{1}\right)\right)\right)$. This yields $a \tilde{x} b \neq c \tilde{x} d$, that is, $a \otimes b-c \otimes d \notin \operatorname{Ker} h$. The theorem is proved.

Let $\mathscr{A}$ be an involutive algebra.

Definition $2.5[8,9]$. The elements $f, f^{*} \in d_{1}(\mathscr{A})$ are called conjugate if

$$
f(x)=\sum_{i=1}^{n} a_{i} \cdot x \cdot b_{i}, \quad f^{*}(x)=\sum_{i=1}^{n} b_{i}^{*} \cdot x \cdot a_{i}^{*} .
$$

Operation $f \leftrightarrow f^{*}$ is an involution for $d_{1}(\mathscr{A})$. It is clear that $f^{*}\left(x^{*}\right)=(f(x))^{*}$ for all $x \in \mathscr{A}$. 
Definition $2.6[8,9]$. An element $f \in d_{1}(\mathscr{A})$ is called self-conjugate if $f=f^{*}$.

Obviously, for self-conjugate $f \in d_{1}(\mathscr{A})$ and for all $x \in \mathscr{A}$ one gets $f\left(x^{*}\right)=(f(x))^{*}$. In addition $f \in d_{1}(\mathscr{A})$ is self-conjugate if and only if for all $i \in\{1,2, \ldots, n\}$ either $b_{i}=a_{i}^{*}$ or there exists $j \in\{1,2, \ldots, n\}, j \neq i$ such that $a_{j}=b_{i}^{*}, b_{j}=a_{i}^{*}$.

Definition $2.7[8,9]$. Self-conjugate $f \in d_{1}(\mathscr{A})$ is called positive if

$$
f(x)=\sum_{i=1}^{n} a_{i} \cdot x \cdot a_{i}^{*}, \quad \text { for some } a_{1}, a_{2}, \ldots, a_{n} \in \mathscr{A} .
$$

This notation is chosen since for $C^{*}$-algebra $\mathscr{A}$ a positive map sends positive elements of algebra $\mathscr{A}$ to positive ones. Indeed, for $C^{*}$-algebra $\mathscr{A}$ one of definitions of positive elements is $x=y y^{*}$ for a certain $y \in \mathscr{A}$. If $f \in d_{1}(\mathscr{A})$ is positive and $x \in \mathscr{A}$ is positive, then

$$
f(x)=\sum_{i=1}^{n} a_{i} \cdot x \cdot a_{i}^{*}=\sum_{i=1}^{n} a_{i} \cdot\left(y y^{*}\right) \cdot a_{i}^{*}=\sum_{i=1}^{n}\left(a_{i} y\right) \cdot\left(y^{*} a_{i}^{*}\right)=\sum_{i=1}^{n}\left(a_{i} y\right) \cdot\left(a_{i} y\right)^{*} .
$$

Since for $C^{*}$-algebra the cone $P$ of positive elements is convex, then $f(x) \in P$.

Now consider some properties of eigenvalues and eigenelements for self-conjugate and positive 1-degree maps. It is useful to note that characteristic values of self-conjugate maps are real. The next three theorems were announced in [9].

Theorem 2.8. If $\mathscr{A}$ is a $C^{*}$-algebra, $f \in d_{1}(\mathscr{A})$ is a positive map, $\lambda \in R$ is a characteristic value of $f$, and there exists a positive characteristic element $x \in \mathscr{A}$, corresponding to the characteristic value $\lambda$, then $\lambda \geq 0$.

Proof. Suppose that $\mathscr{A}$ is a $C^{*}$-algebra, $f \in d_{1}(\mathscr{A})$ is a positive map, $x \in \mathscr{A}$ is a positive element $(x \neq 0), \lambda \in R, f(x)=\lambda x$. If $\lambda<0$, then $-\lambda>0$ and $-\lambda x$ is positive, that is, $\lambda x \in(-P)$, where $P$ is the cone of positive elements. But $\lambda x=f(x) \in P$. Hence $\lambda x \in$ $P \cap(-P)=\{0\}$. It is impossible since for $\lambda \neq 0, x \neq 0, \lambda x \neq 0$. Therefore the assumption $\lambda<0$ is false and $\lambda \geq 0$.

Theorem 2.9. If $\mathscr{A}$ is an involutive algebra, $f \in d_{1}(\mathscr{A})$ is a self-conjugate map, $\lambda \in R$ is a characteristic value of $f$, then there exists a self-conjugate characteristic element $x \in \mathscr{A}(x \neq$ $0)$ corresponding to the characteristic value $\lambda$.

Proof. For any $x \in \mathscr{A}$ there exists a unique representation as the sum of "real" and "imaginary" parts $x=x_{1}+i x_{2}$, where $x_{1}, x_{2} \in \mathscr{A}$ are self-conjugate elements $\left(x_{1}=(1 / 2)(x+\right.$ $\left.\left.x^{*}\right), x_{2}=(1 / 2 i)\left(x-x^{*}\right)\right)$. Therefore $f(x)=\lambda x \Leftrightarrow f\left(x_{1}+i x_{2}\right)=\lambda\left(x_{1}+i x_{2}\right) \Leftrightarrow f\left(x_{1}\right)+i f\left(x_{2}\right)=$ $\lambda x_{1}+i \lambda x_{2}$.

The elements $\lambda x_{1}, \lambda x_{2}$ are self-conjugate. As $f, x_{1}, x_{2}$ are self-conjugate, $f\left(x_{1}\right), f\left(x_{2}\right)$ are self-conjugate as well. Since the representation as the sum of "real" and "imaginary" 
parts is unique, then $f\left(x_{1}\right)=\lambda x_{1}, f\left(x_{2}\right)=\lambda x_{2}$. For $x \neq 0$ at least one element $x_{1}$ or $x_{2}$ is not equal to zero. This completes the proof.

Theorem 2.10. If $\mathscr{A}$ is a $C^{*}$-algebra, $f \in d_{1}(\mathscr{A})$ is a positive map, and there is a characteristic value $\lambda<0$ of $f$, then there exist positive elements $x_{1}, x_{2}, a \in \mathcal{A}$ such that $f\left(x_{1}\right)-\lambda x_{1}=$ $f\left(x_{2}\right)-\lambda x_{2}=a$.

Proof. A positive map is self-conjugate. From Theorem 2.9 it follows that there is a selfconjugate element $x \in \mathscr{A}$ such that $x \neq 0$ and $f(x)=\lambda x$. If $\mathscr{A}$ is $C^{*}$-algebra and $x \in \mathscr{A}$ is a self-conjugate element, then there exists unique couple of positive or equal to zero elements $x_{1}, x_{2} \in \mathscr{A}$ such that $x=x_{1}-x_{2}, x_{1} x_{2}=x_{2} x_{1}=0$.

We have $f(x)=f\left(x_{1}-x_{2}\right)=\lambda\left(x_{1}-x_{2}\right)=\lambda x_{1}-\lambda x_{2}$. Therefore $f\left(x_{1}\right)-\lambda x_{1}=f\left(x_{2}\right)-$ $\lambda x_{2}$. Denote this element by $a$. It follows from Theorem 2.8 that $x_{1} \neq 0, x_{2} \neq 0$. Hence both $x_{1}$ and $x_{2}$ are positive. As $x_{1}, x_{2}, f,(-\lambda)$ are positive, then $f\left(x_{1}\right), f\left(x_{2}\right),\left(-\lambda x_{1}\right)$, $\left(-\lambda x_{2}\right)$, and $a$ are positive. This completes the proof.

If $\mathscr{A}$ is a normed algebra, then the standard norm on $d_{1}(\mathscr{A})$ is also given, since $d_{1}(\mathscr{A}) \subset$ $L(A)$, that is, for $f \in d_{1}(\mathscr{A})$,

$$
\|f\|=\sup _{x \neq 0} \frac{\|f(x)\|}{\|x\|} .
$$

Obviously, $\left\|f^{*}\right\|=\|f\|$ and if $f(x)=\sum_{i=1}^{n} a_{i} \cdot x \cdot b_{i}$, then $\|f\| \leq \sum_{i=1}^{n}\left\|a_{i}\right\| \cdot\left\|b_{i}\right\|$.

As far as $L(A)$ is closed, $\overline{d_{1}(\mathscr{A})} \subset L(A)$, where $\overline{d_{1}(\mathscr{A})}$ is the closure of $d_{1}(\mathscr{A})$.

Definition 2.11. A normed algebra $\mathscr{A}$ is $\overline{1}$-algebra if $\overline{d_{1}(\mathscr{A})}=L(A)$. Obviously, if $\mathscr{A}$ is a normed 1-algebra, then $\mathscr{A}$ is a $\overline{1}$-algebra.

Definition 2.12. If $\mathscr{A}$ is an involutive algebra, then the maps $f, f^{*} \in \overline{d_{1}(\mathscr{A})}$ are called conjugate if $f(x)=\lim _{n \rightarrow \infty} f_{n}(x), f^{*}(x)=\lim _{n \rightarrow \infty} f_{n}^{*}(x)$ for some $f_{1}, f_{2}, \ldots, f_{n}, \ldots \in d_{1}(\mathscr{A})$.

\section{1-degree maps of modules}

(A) Let $M_{1}, M_{2}$ be free finitely generated $\mathscr{A}$-modules,

$M_{1}=\mathscr{A}^{n}, \quad M_{2}=\mathscr{A}^{m}, \quad F: M_{1} \longrightarrow M_{2}, \quad F=\left(\begin{array}{c}f_{1} \\ f_{2} \\ \vdots \\ f_{m}\end{array}\right), \quad f_{i}=P_{i} \circ F: M_{1} \longrightarrow \mathscr{A}$,

where $P_{i}: M_{2} \rightarrow \mathscr{A}$ is the projection of $M_{2}=\mathscr{A} \oplus \mathscr{A} \oplus \cdots \oplus \mathscr{A}$ onto ith term.

Definition 3.1. $F: M_{1} \rightarrow M_{2}$ is called 1-degree (1-degree) map if for each $i \in\{1,2, \ldots, m\}$ the relation $f_{i}\left(x_{1}, x_{2}, \ldots, x_{n}\right)=f_{i 1}\left(x_{1}\right)+f_{i 2}\left(x_{2}\right)+\cdots+f_{\text {in }}\left(x_{n}\right)$ holds, where $f_{i 1}, f_{i 2}, \ldots, f_{\text {in }} \in$ $d_{1}(\mathscr{A})\left(\in \overline{d_{1}(\mathscr{A})}\right)$. 
Assign the following matrix to a 1-degree or a $\overline{1}$-degree map:

$$
F \longleftrightarrow\left(\begin{array}{cccc}
f_{11} & f_{12} & \cdots & f_{1 n} \\
f_{21} & f_{22} & \cdots & f_{2 n} \\
\vdots & \vdots & & \vdots \\
f_{m 1} & f_{m 2} & \cdots & f_{m n}
\end{array}\right) .
$$

Definition 3.2. If $\mathscr{A}$ is an involutive algebra, then the 1-degree or $\overline{1}$-degree maps $F=$ $\left(f_{i j}\right)_{m \times n}, F^{*}=\left(f_{i j}^{*}\right)_{m \times n}$ are called conjugate.

As well as it is for algebra maps, $F^{*}\left(x^{*}\right)=(F(x))^{*}$.

If the modules $M_{1}, M_{2}$ are normed (e.g., for a normed algebra $\mathscr{A}$ the norm can be defined as $\|x\|=\sqrt{\sum_{i}\left\|x_{i}\right\|^{2}}$ ), then $\|F\|$ is defined in the standard way

$$
\|F\|=\sup _{x \neq 0} \frac{\|F(x)\|}{\|x\|} .
$$

The straightforward computation gives

$$
\|F\| \leq \sqrt{\sum_{i=1}^{m}\left(\sum_{j=1}^{n}\left\|f_{i j}\right\|\right)^{2}} \leq \sqrt{\sum_{i=1}^{m} \sum_{j=1}^{n}\left\|f_{i j}\right\|^{2}}
$$

and $\|F\|=\left\|F^{*}\right\|$.

(B) Let $\mathscr{A}$ be a $C^{*}$-algebra and let $l_{2}(\mathscr{A})$ be a Hilbert $\mathscr{A}$-module,

$$
F: l_{2}(\mathscr{A}) \longrightarrow l_{2}(\mathscr{A}), \quad F=\left(\begin{array}{c}
f_{1} \\
f_{2} \\
\vdots
\end{array}\right),
$$

where $f_{i}=P_{i} \circ F$, as above. Since $F(x) \in l_{2}(\mathscr{A})$ it has to be $\|F(x)\|^{2}=\sum_{i=1}^{\infty}\left\|f_{i}(x)\right\|^{2}<\infty$ for any $x \in l_{2}(\mathscr{A})$. This is fulfilled, in particular, if $\sum_{i=1}^{\infty}\left\|f_{i}\right\|^{2}<\infty$. In this case

$$
\|F(x)\|^{2} \leq \sum_{i=1}^{\infty}\left\|f_{i}\right\|^{2} \cdot\|x\|^{2}
$$

therefore

$$
\|F\| \leq \sqrt{\sum_{i=1}^{\infty}\left\|f_{i}\right\|^{2}}<\infty \quad \text { for }\|F\|=\sup _{x \neq 0} \frac{\|F(x)\|}{\|x\|} .
$$

Definition 3.3. A bounded map $F: l_{2}(\mathscr{A}) \rightarrow l_{2}(\mathscr{A})$ is called 1-degree map if for each $i \in$ $N f_{i}(x)=f_{i}\left(x_{1}, x_{2}, \ldots\right)=\sum_{j=1}^{\infty} f_{i j}\left(x_{j}\right)$, where $f_{i j} \in d_{1}(\mathscr{A})$ for any $j \in N$,

$$
F \longleftrightarrow\left(\begin{array}{ccc}
f_{11} & f_{12} & \cdots \\
f_{21} & f_{22} & \cdots \\
\vdots & \vdots & \ddots
\end{array}\right)
$$


Since $\lim _{n \rightarrow \infty} \sum_{j=1}^{n} f_{i j}\left(x_{j}\right)$ must exist for all $x=\left(x_{1}, x_{2}, \ldots\right) \in l_{2}(\mathscr{A})$, we assume that for each $i \in N$ there exists a constant $M_{F, i}>0$ such that $\left\|f_{i j}\right\| \leq M_{F, i}$ for each $j \in N$. Then

$$
\begin{aligned}
\left\|f_{i}(x)\right\|^{2} & =\left\|\sum_{j=1}^{\infty} f_{i j}\left(x_{j}\right)\right\|^{2} \leq \sum_{j=1}^{\infty}\left\|f_{i j}\right\|^{2} \cdot\left\|x_{j}\right\|^{2} \\
& \leq \sum_{j=1}^{\infty} M_{F, i}^{2} \cdot\left\|x_{j}\right\|^{2}=M_{F, i}^{2} \sum_{j=1}^{\infty}\left\|x_{j}\right\|^{2}
\end{aligned}
$$

and $\left\|f_{i}\right\| \leq M_{F, i}$. Assume that $\sum_{i=1}^{\infty} M_{F, i}^{2}<\infty$ for each 1-degree map $F$. Then for each 1degree map $F\|F\|<\infty$.

By $d_{1}\left(l_{2}(\mathscr{A})\right)$ denote the set of all 1-degree maps from $l_{2}(\mathscr{A})$ to $l_{2}(\mathscr{A})$ and by $\overline{d_{1}\left(l_{2}(\mathscr{A})\right)}$ denote its closure. We say that $F: l_{2}(\mathscr{A}) \rightarrow l_{2}(\mathscr{A})$ is a $\overline{1}$-degree map if $F \in \overline{d_{1}\left(l_{2}(\mathscr{A})\right)}$. Obviously, $F \in \overline{d_{1}\left(l_{2}(\mathscr{A})\right)} \leftrightarrow f_{i j} \in \overline{d_{1}(\mathscr{A})}$ for all $i, j \in N$.

Let $e_{1}=(1,0,0, \ldots), e_{2}=(0,1,0, \ldots), e_{3}=(0,0,1, \ldots)$, and so forth be basic elements in $l_{2}(\mathscr{A})$ and let $L_{n, \mathscr{A}}^{\perp}$ be a submodule of the module $l_{2}(\mathscr{A})$ generated by elements $e_{n+1}, e_{n+2}, \ldots$

Definition 3.4. Following [14], we say that a map $F \in \overline{d_{1}\left(l_{2}(\mathscr{A})\right)}$ is compact if $\lim _{n \rightarrow \infty}$ $\left\|\left.F\right|_{L_{n, \mathcal{A}}^{\perp}}\right\|=0$.

Definition 3.5. A map $F \in \overline{d_{1}\left(l_{2}(\mathscr{A})\right)}$ is called Fredholm $\overline{1}$-map if $F=H+C$, where $H, C \in$ $\overline{d_{1}\left(l_{2}(\mathscr{A})\right)}, H$ is an isomorphism, and $C$ is a compact map.

The set $C_{\overline{1}}\left(l_{2}(\mathscr{A})\right)$ of all compact $\overline{1}$-degree maps from $l_{2}(\mathscr{A})$ to $l_{2}(\mathscr{A})$ is an ideal in the algebra $\overline{d_{1}\left(l_{2}(\mathscr{A})\right)}$.

Definition 3.6. A manifold $M$ modelled by $l_{2}(\mathscr{A})$ is called Fredholm $\overline{1}$-manifold if $M$ has an atlas with transformation functions having the "derivatives" in the form $I+C$, where $I$ is the identity map, $C \in C_{\overline{1}}\left(l_{2}(\mathscr{A})\right)$.

By $\mathrm{GL}_{\overline{1}}\left(l_{2}(\mathscr{A})\right)$ denote the group of $\overline{1}$-degree isomorphisms of $l_{2}(\mathscr{A})$.

Theorem 3.7. If $\mathscr{A}$ is $C^{*}-\overline{1}$-algebra, then $\mathrm{GL}_{\overline{1}}\left(l_{2}(\mathscr{A})\right)$ is contractible.

Proof. Let $E$ be the Banach space obtained from $l_{2}(\mathscr{A})$ by ignoring the element multiplication in $\mathscr{A}$, where $\|\cdot\|_{E}=\|\cdot\|_{l_{2}(\mathscr{A})}$.

For $C^{*}-\overline{1}$-algebra $\mathscr{A}$ we get $\overline{d_{1}\left(l_{2}(\mathscr{A})\right)}=B(E)$ and $\mathrm{GL}_{\overline{1}}\left(l_{2}(\mathscr{A})\right)=\mathrm{GL}(E)$. Hence $\mathrm{GL}_{\overline{1}}$ $\left(l_{2}(\mathscr{A})\right)$ is an open subset of Banach space $B(E)$. Therefore, by Milnor's theorem [13] $\mathrm{GL}_{\overline{1}}\left(l_{2}(\mathscr{A})\right)$ has the homotopy type of a $C W$-complex and by Whitehead's theorem [16] the strong and weak homotopy trivialities of $\mathrm{GL}_{\overline{1}}\left(l_{2}(\mathscr{A})\right)$ are equivalent. So, it is sufficient to prove the weak homotopy triviality.

The proof technique repeats that for $\mathrm{GL}(H)$ in [11] or for $\mathrm{GL}\left(l_{2}(\mathscr{A})\right)$ in [12]. First we use Atiyah's theorem on small balls: if $f$ is a continuous map from $n$-dimensional sphere $S^{n}$ to $\mathrm{GL}_{\overline{1}}\left(l_{2}(\mathscr{A})\right)$, then $f$ is homotopic to $f^{\prime}$ so that $f^{\prime}\left(S^{n}\right)$ is a finite polyhedron lying in $\mathrm{GL}_{\overline{1}}\left(l_{2}(\mathscr{A})\right)$ together with the homotopy.

Then we use from [11, Lemma 3]: there exist a decomposition $l_{2}(\mathscr{A})$ in $H^{\prime} \oplus H_{1}$, where $H^{\prime} \cong H_{1} \cong l_{2}(\mathscr{A})$, and a continuous map $f^{\prime \prime}: S^{n} \rightarrow \mathrm{GL}_{\overline{1}}\left(l_{2}(\mathscr{A})\right)$ homotopic to $f^{\prime}$ such that $f^{\prime \prime}(s)(x)=x$ for all $s \in S^{n}, x \in H^{\prime}$. 
Next we prove the statement similar to [11, Lemma 7], and [12, Lemma 7.1.4]: $V=$ $\left\{g \in \mathrm{GL}_{\overline{1}}\left(l_{2}(\mathscr{A})\right)|g|_{H^{\prime}}=I d_{H^{\prime}}, g\left(H_{1}\right)=H_{1}\right\}$ is contractible to 1 in $\mathrm{GL}_{\overline{1}}\left(l_{2}(\mathscr{A})\right)$. Here we use the condition from the hypothesis of our theorem that $\mathscr{A}$ is $C^{*}-\overline{1}$-algebra. If so, then for any $u \in \mathrm{GL}_{\overline{1}}\left(l_{2}(\mathscr{A})\right), u^{-1}$ and all linear combinations of $u$ and $u^{-1}$ belong to $\mathrm{GL}_{\overline{1}}\left(l_{2}(\mathscr{A})\right)$ as well. The construction of homotopy is the same as in $[11,12]$.

Since $H^{\prime} \cong l_{2}(\mathscr{A})$, we can decompose $H^{\prime}$ into the sum $H^{\prime}=H_{2} \oplus H_{3} \oplus \cdots H_{i} \cong l_{2}(\mathscr{A})$, then we get $l_{2}(\mathscr{A})=H_{1} \oplus H_{2} \oplus H_{3} \oplus \cdots$.

Let $g \in V$ on, then the matrix of $g$ related to the above decomposition is $g=\operatorname{diag}(u, 1$, $1,1, \ldots)=\operatorname{diag}\left(u, u^{-1} u, 1, u^{-1} u, 1, \ldots\right)$, where $u=\left.g\right|_{H_{1}}$.

For $t \in[0, \pi / 2]$ we get $\left.g_{t}\right|_{H_{1}}=\left.g\right|_{H_{1}}=u$ and for each $i \in N$,

$$
\begin{aligned}
\left.g_{t}\right|_{H_{2 i} \oplus H_{2 i+1}} & =\left(\begin{array}{cc}
\cos t & -\sin t \\
\sin t & \cos t
\end{array}\right)\left(\begin{array}{ll}
u & 0 \\
0 & 1
\end{array}\right)\left(\begin{array}{cc}
\cos t & \sin t \\
-\sin t & \cos t
\end{array}\right)\left(\begin{array}{cc}
u^{-1} & 0 \\
0 & 1
\end{array}\right) \\
& =\left(\begin{array}{cc}
1 \cdot \cos ^{2} t+u^{-1} \sin ^{2} t & (u-1) \sin t \cos t \\
\left(1-u^{-1}\right) \sin t \cos t & u \sin ^{2} t+1 \cdot \cos ^{2} t
\end{array}\right) .
\end{aligned}
$$

For $t \in[\pi / 2, \pi]$ and for each $i \in N$

$$
\begin{aligned}
\left.g_{t}\right|_{H_{2 i-1} \oplus H_{2 i}} & =\left(\begin{array}{cc}
\cos t & -\sin t \\
\sin t & \cos t
\end{array}\right)\left(\begin{array}{cc}
u^{-1} & 0 \\
0 & 1
\end{array}\right)\left(\begin{array}{cc}
\cos t & \sin t \\
-\sin t & \cos t
\end{array}\right)\left(\begin{array}{ll}
u & 0 \\
0 & 1
\end{array}\right) \\
& =\left(\begin{array}{cc}
1 \cdot \cos ^{2} t+u \sin ^{2} t & \left(u^{-1}-1\right) \sin t \cos t \\
(1-u) \sin t \cos t & u^{-1} \sin ^{2} t+1 \cdot \cos ^{2} t
\end{array}\right),
\end{aligned}
$$

$g_{0}=g, g_{\pi / 2}=\operatorname{diag}\left(u, u^{-1}, u, u^{-1}, u, \ldots\right)$ by both formulas of homotopies, and $g_{\pi}=\operatorname{diag}(1$, $1,1,1, \ldots)$.

Since all these maps are linear isomorphisms in $L(E)$, then by the hypothesis of the theorem they are $\overline{1}$-degree isomorphisms.

At last we repeat [12, Lemma 7.1.5]: the set

$$
W=\left\{g \in \mathrm{GL}_{\overline{1}}\left(l_{2}(\mathscr{A})\right)|g|_{H^{\prime}}=I d_{H^{\prime}}\right\}
$$

is contractible to $V$. (In [12] this is proved for $\operatorname{GL}\left(l_{2}(\mathscr{A})\right)$.) This concludes the proof.

\section{Polynomials over a noncommutative algebra and nonlinear Fredholm maps}

Let $\mathscr{A}$ be a noncommutative algebra, $x \notin \mathscr{A}, x^{1}=x, x^{k} \cdot x^{l}=x^{k+l}$ for all $k, l \in N$. Let us give the recurrent definition of a monomial in $x$.

Definition 4.1. (1) Every element of $\mathscr{A}$ is a 0 -degree monomial.

(2) $x$ is a 1-degree monomial.

(3) If $m_{1}$ is a $k$-degree monomial for any $k \in N$ and $m_{2}$ is an $l$-degree monomial for any $l \in N$, then $m_{1} \cdot m_{2}$ is a $(k+l)$-degree monomial.

(4) The other monomials do not exist.

Definition 4.2. A finite sum of $n$-degree monomials is called a homogeneous n-degree polynomial. 
Definition 4.3. A finite sum of homogeneous polynomials is called an all linearn-degree polynomial, where $n$ is the maximal degree of the terms.

The set $P$ of all polynomials forms a graded algebra $P=\oplus_{n=1}^{\infty} P_{n}$, where $P_{n}$ is the set of homogeneous $n$-degree polynomials. Obviously, $P_{0} \cong \mathscr{A}, P_{1} \cong \mathscr{A} \otimes \widetilde{A}$.

Definition 4.4. The map corresponding to the homogeneous $n$-degree polynomial is called an $n$-degree map and the map corresponding to any polynomial is called a polynomial map.

The set $d(\mathscr{A})$ of all polynomial maps forms a graded algebra as well: $d(\mathscr{A})=\oplus_{n=1}^{\infty} d_{n}(\mathscr{A})$, where $d_{n}(\mathscr{A})$ is the set of $n$-degree maps. There is a natural map of graded algebras $h: P \rightarrow d(\mathscr{A})$ assigning to the polynomial its corresponding map. For $n=1$ it was shown in Section 2 that $\left.h\right|_{P_{1}}: P_{1} \cong \mathscr{A} \otimes \widetilde{\mathscr{A}} \rightarrow d_{1}(\mathscr{A})$ is not injective. Polynomial maps defined above are maps that have 1-degree "derivatives."

We will obtain the definition of a monomial in several variables $x_{1}, x_{2}, \ldots, x_{n}, \ldots$ if in Definition 4.1 we replace (2) by " $x_{i}$ is a 1-degree monomial for every $i \in N$." Then Definitions 4.2 and 4.3 give the notions of polynomials in $x_{1}, x_{2}, \ldots, x_{n}, \ldots$ and of corresponding polynomial maps from $l_{2}(\mathscr{A})$ to $\mathscr{A}$.

Definition 4.5. The map $F: U \rightarrow l_{2}(\mathscr{A})$ is called polynomial if $f_{i}$ are polynomial maps for all $i \in N$, where $U \subset l_{2}(\mathscr{A})$ is an open domain and

$$
F=\left(\begin{array}{c}
f_{1} \\
f_{2} \\
\vdots
\end{array}\right) .
$$

The "derivative"

$$
F^{\prime}(x)=\left(\begin{array}{c}
d f_{1} \\
d f_{2} \\
\vdots
\end{array}\right)
$$

of a polynomial map $F$ is a 1-degree map for every $x \in U$.

Definition 4.6. The polynomial map $F: U \rightarrow l_{2}(\mathscr{A})$ is called a Fredholm map if $F^{\prime}(x)$ is a Fredholm 1-degree map for each $x \in U$.

\section{A certain application of 1 -degree maps}

In probability theory a discrete Markov chain is described by the transition matrix consisting of elements from $[0,1]$. Markov's theorem proclaims that if some power of this matrix does not contain zeroes, then limit transition probabilities exist. We assume that $k$ th power of the transition matrix does not contain zeroes and so the question is arising: how the elements of transition matrix that can be changed for $k$ th power of matrix remain without zeroes.

Assume that the transition matrix is an $n \times n$-matrix and consider the set of the real $n \times n$-matrices as a normed involutive algebra with transposition as the involution and 
the norm $\|A\|=n \cdot \max _{1 \leq i, j \leq n}\left|a_{i j}\right|$, (equivalent to the norm $\|A\|=\sup _{x \neq 0}\|A x\| /\|x\|$ but more suitable for the calculation). The inequality $\|A B\| \leq\|A\| \cdot\|B\|$ for the normed algebra is fulfilled.

Let $P=\left(p_{i j}\right)_{n \times n}$ be a transition matrix, let $P^{k}=\left(p_{i j}^{(k)}\right)_{n \times n}$ be the $k$ th power of $P$ and $f(P)=P^{k}$. Using the equality $f(P+\Delta P)-f(P)=(P+\Delta P)^{k}-P^{k}=d f+o(\Delta P)$ we approximate the difference at the left-hand side by the "differential" $d f$ and instead of the inequality $\|\Delta f\|=\|f(P+\Delta P)-f(P)\|<\varepsilon$ we solve the inequality $\|d f\|<\varepsilon$. Here

$$
d f=P^{k-1} \cdot \Delta P+P^{k-2} \cdot \Delta P \cdot P+\cdots+P \cdot \Delta P \cdot P^{k-2}+\Delta P \cdot P^{k-1}
$$

is a $d_{1}$-map of $\Delta P$,

$$
\begin{aligned}
o(\Delta P)= & {\left[P^{k-2} \cdot(\Delta P)^{2}+P^{k-3} \cdot(\Delta P)^{2} \cdot P+\cdots+P \cdot(\Delta P)^{2} \cdot P^{k-3}\right.} \\
& \left.+P^{k-3} \cdot \Delta P \cdot P \cdot \Delta P+\cdots+\Delta P \cdot P \cdot \Delta P \cdot P^{k-3}\right] \\
& +\left[P^{k-3} \cdot(\Delta P)^{3}+\cdots\right]+\cdots+(\Delta P)^{k} .
\end{aligned}
$$

Taking into account the estimate

$$
\begin{aligned}
\|d f\| \leq & \left\|P^{k-1}\right\| \cdot\|\Delta P\|+\left\|P^{k-2}\right\| \cdot\|\Delta P\| \cdot\|P\| \\
& +\cdots+\|P\| \cdot\|\Delta P\| \cdot\left\|P^{k-2}\right\|+\|\Delta P\| \cdot\left\|P^{k-1}\right\| \\
\leq & \|P\|^{k-1} \cdot\|\Delta P\|+\|P\|^{k-2} \cdot\|\Delta P\| \cdot\|P\| \\
& +\cdots+\|P\| \cdot\|\Delta P\| \cdot\left\|P^{k-2}\right\|+\|\Delta P\| \cdot\left\|P^{k-1}\right\|=k \cdot\|P\|^{k-1} \cdot\|\Delta P\|,
\end{aligned}
$$

it is sufficient to solve the inequality $k \cdot\|P\|^{k-1} \cdot\|\Delta P\|<\varepsilon$. Thus we get

$$
\|\Delta P\|<\delta_{1}(\varepsilon)=\frac{\varepsilon}{k \cdot\|P\|^{k-1}},
$$

therefore for all $i, j \in\{1,2, \ldots, n\}$,

$$
\left|\Delta p_{i j}\right|<\frac{\varepsilon}{n \cdot k \cdot\|P\|^{k-1}} .
$$

Further we will not reject $o(\Delta P)$ and obtain more exact estimate:

$$
\begin{aligned}
\|o(\Delta P)\| \leq & {\left[\|P\|^{k-2} \cdot\|\Delta P\|^{2}+\|P\|^{k-3} \cdot\|\Delta P\|^{2} \cdot\|P\|+\cdots\right.} \\
& +\|P\| \cdot\|\Delta P\|^{2} \cdot\|P\|^{k-3}+\|\Delta P\|^{2} \cdot\|P\|^{k-2} \\
& \left.+\|P\|^{k-3} \cdot\|\Delta P\| \cdot\|P\| \cdot\|\Delta P\|+\cdots+\|\Delta P\| \cdot\|P\| \cdot\|\Delta P\| \cdot\|P\|^{k-3}\right] \\
& +\left[\|P\|^{k-3} \cdot\|\Delta P\|^{3}+\cdots\right]+\cdots+\|\Delta P\|^{k} \\
= & C_{k}^{2} \cdot\left\|P^{k-2} \cdot\right\| \Delta P\left\|^{2}+C_{k}^{3} \cdot\right\| P\left\|^{k-3} \cdot\right\| \Delta P\left\|^{3}+\cdots+C_{k}^{s} \cdot\right\| P\left\|^{k-s} \cdot\right\| \Delta P \|^{s} \\
& +\cdots+\|\Delta P\|^{k}=(\|P\|+\|\Delta P\|)^{k}-\|P\|^{k}-k \cdot\|P\|^{k-1} \cdot\|\Delta P\| .
\end{aligned}
$$


Let $\varphi(x)=x^{k}$ be a real function. Then for $x=\|P\|, \Delta x=\|\Delta P\|$ we get

$$
\begin{gathered}
\Delta \varphi=\varphi(x+\Delta x)-\varphi(x)=\varphi(\|P\|+\|\Delta P\|)-\varphi(\|P\|)=(\|P\|+\|\Delta P\|)^{k}-\|P\|^{k}, \\
d \varphi=\varphi^{\prime}(x) \Delta x=k x^{k-1} \Delta x=k \cdot\|P\|^{k-1} \cdot\|\Delta P\|, \\
(\|P\|+\|\Delta P\|)^{k}-\|P\|^{k}-k \cdot\|P\|^{k-1} \cdot\|\Delta P\|=\Delta \varphi-d \varphi=o(\Delta x)=o(\|\Delta P\|) .
\end{gathered}
$$

Consider $o(\Delta x)$ in the form $\left(\varphi^{\prime \prime}(x+t \cdot \Delta x) / 2\right)(\Delta x)^{2}$, where $0<t<1$. For $\varphi^{\prime \prime}(x)=k(k-$ 1) $x^{k-2}$, we obtain $\varphi^{\prime \prime}(x+t \cdot \Delta x)=k(k-1)(x+t \cdot \Delta x)^{k-2}$.

Since $P$ and $P+\Delta P$ are transition matrices, that is, their elements are probabilities, then $\left|\Delta p_{i j}\right| \leq 1$ for all $i, j \in\{1,2, \ldots, n\}$ and $\|\Delta P\| \leq n$. Thus for $x=\|P\|, \Delta x=\|\Delta P\|$ we get $x \geq 0,0<\Delta x \leq n, x+t \cdot \Delta x \leq x+\Delta x \leq x+n$, and $(x+t \cdot \Delta x)^{k-2} \leq(x+n)^{k-2}$. Therefore $\|o(\Delta P)\| \leq(k(k-1) / 2)(\|P\|+n)^{k-2}\|\Delta P\|^{2}$ and

$$
\left\|(P+\Delta P)^{k}-P^{k}\right\| \leq\|d f\|+\|o(\Delta P)\| \leq k \cdot\|P\|^{k-1} \cdot\|\Delta P\|+\frac{k(k-1)}{2}(\|P\|+n)^{k-2}\|\Delta P\|^{2} .
$$

Now we have to solve the square inequality

$$
k \cdot\|P\|^{k-1} \cdot\|\Delta P\|+\frac{k(k-1)}{2}(\|P\|+n)^{k-2}\|\Delta P\|^{2}<\varepsilon
$$

for $\|\Delta P\|$. Taking into account $\|\Delta P\| \geq 0$ we obtain

$$
\|\Delta P\|<\delta_{2}(\varepsilon)=\frac{\sqrt{\left(k\|P\|^{k-1}\right)^{2}+2 \varepsilon k(k-1)(\|P\|+n)^{k-2}}-k\|P\|^{k-1}}{k(k-1)(\|P\|+n)^{k-2}} .
$$

Since $\|\Delta P\|=n \cdot \max _{1 \leq i, j \leq n}\left|\Delta p_{i j}\right|$, we get for all $i, j \in\{1,2, \ldots, n\}$,

$$
\left|\Delta p_{i j}\right|<\frac{\sqrt{\left(k\|P\|^{k-1}\right)^{2}+2 \varepsilon k(k-1)(\|P\|+n)^{k-2}}-k\|P\|^{k-1}}{n k(k-1)(\|P\|+n)^{k-2}} .
$$

This is a sufficient condition for $\|\Delta f\|<\varepsilon$.

The number $\varepsilon$ must be chosen so that all elements of the matrix $(P+\Delta P)^{k}$ are strictly positive, that is, they differ from the corresponding elements of $P^{k}$ less than by $m=$ $\min _{1 \leq i, j \leq n} p_{i j}^{(k)}>0$. Hence it must be $\|d f\|=\left\|(P+\Delta P)^{k}-P^{k}\right\|<n m$, therefore $\varepsilon$ has to be chosen so that $\varepsilon \leq n m$, for example, $\varepsilon=n m$.

In this case the first formula yields $\left|\Delta p_{i j}\right|<m / k\|P\|^{k-1}$ and the second formula yields

$$
\left|\Delta p_{i j}\right|<\frac{\sqrt{\left(k\|P\|^{k-1}\right)^{2}+2 n m k(k-1)(\|P\|+n)^{k-2}}-k\|P\|^{k-1}}{n k(k-1)(\|P\|+n)^{k-2}}
$$

for all $i, j \in\{1,2, \ldots, n\}$. The formulae (5.5) and (5.12) were announced in [10]. 


\section{Conclusion}

Let us ascertain the interdependence between the notions defined in this paper and some other ones.

(1) Let $f$ be a polynomial in $x$ with coefficients from $\mathscr{A}$, then $d f$ is a 1-degree polynomial in $\Delta x$, where the coefficients are also polynomials in $x$. So, $d: P \rightarrow P \otimes \widetilde{P}$ (see Section 2 for the description of what $\widetilde{\mathscr{A}}$ with respect to $\mathscr{A}$ is). Let $g: P \otimes \widetilde{P} \rightarrow P$ be an algebra homomorphism defined as $g\left(f_{1} \otimes f_{2}\right)=f_{1} \cdot f_{2}$. Then $\delta=g \circ d: P \rightarrow P$ is a derivation of the algebra $P$, that is, $\delta\left(f_{1} \cdot f_{2}\right)=\delta\left(f_{1}\right) \cdot f_{2}+f_{1} \cdot \delta\left(f_{2}\right)$ for all $f_{1}, f_{2} \in P$. If $f$ is an $n$-degree polynomial, then $\delta(f)$ is also an $(n-1)$-degree polynomial.

(2) For a noncommutative operator algebra $\mathscr{A}$ consider the algebra homomorphisms $\mu_{A}: \mathscr{F}_{1} \rightarrow \mathscr{A}$ and $\mu_{A_{1}, \ldots, A_{n}}: \mathscr{F}_{n} \rightarrow \mathscr{A}$ that are defined, for example, in [15]. Here $A, A_{1}, \ldots$, $\stackrel{n}{A}_{n} \in \mathscr{A}$ are called generators, $\mathscr{F}_{1}$ is a space of one-place symbols, $\mathscr{F}_{n}$ is a space of $n$ place symbols, numbers over letters are called Feynman numbers, and the set of letters with numbers over them is called Feynman set. The one-place or $n$-place symbols are real or complex functions of one or $n$ variables, respectively. These homomorphisms $\mu_{A}$ and $\mu_{A_{1}, \ldots, A_{n}}$ may be called "evaluation homomorphisms" because they give a possibility to find in $\mathscr{A}$ the values $f(A)$ or $f\left(\stackrel{1}{A}_{1}, \ldots, \stackrel{n}{A}\right.$ ) for different $f$ and specified $A$ or $\stackrel{1}{A}_{1}, \ldots, \stackrel{n}{A}_{n}$. For example, if $f$ is a polynomial in one or several variables, it is necessary to substitute $A$ or $\stackrel{1}{A}_{1}, \ldots, \stackrel{n}{A}_{n}$ for variables of $f$.

If $f$ is a polynomial in $2 n+1$ variables, $f=f\left(y_{1}, \ldots, y_{n}, z, x_{1}, \ldots, x_{n}\right)=\sum_{i=1}^{n} y_{i} \cdot z \cdot x_{i}$,

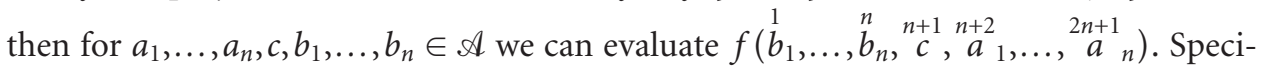
fying $a_{1}, \ldots, a_{n}, b_{1}, \ldots, b_{n}$ and replacing $c$ by $\mathscr{A}$, we obtain the 1 -degree map corresponding to the 1-degree polynomial in $x$ of the form $\sum_{i=1}^{n} a_{i} \cdot x \cdot b_{i}$. Obviously, for another polynomial it is necessary to use another homomorphism and the quantity of generators depends on the quantity of polynomial terms.

\section{References}

[1] O. Bratteli and D. W. Robinson, Operator Algebras and Quantum Statistical Mechanics. Vol. 1, Texts and Monographs in Physics, Springer, New York, 1979.

[2] M. N. Krein, 1-degree maps in non-commutative algebras and integration in them, Operators with Partial Integrals (2000), no. 4, 79-84 (Russian).

[3] _ Differentiation in non-commutative algebras, Nonlinear Analysis and FunctionalDifferential Equations (MNK ADM, 2000). Abstracts of Reports, Voronezh, 2000, p. 135.

[4] - Fredholm GLG-maps in modules over non-commutative algebras, Modern Methods of Function Theory and Adjoined Problems. Abstracts of Reports, Voronezh, 2001, p. 155.

[5] _ On 1-degree maps in noncommutative $C^{*}$-algebras, Voronezh Winter Mathematical School, 2002, Voronezh, 2002, p. 46.

[6] _ Symmetry of 1-degree maps and maps of arbitrary degree in $C^{*}$-algebras, Modern Methods in the Theory of Boundary Value Problems. Proceedings of Voronezh Spring Mathematical School, Voronezh, 2004, pp. 123-124. 
[7] _ On the properties of 1-degree maps in non-commutative algebras, Topological and Variational Methods of Nonlinear Analysis and Their Applications. Abstracts of the Conference, Voronezh, 2005, pp. 67-68.

[8] _ 1-degree maps in non-commutative algebras and modules over them, preprint, 2002.

[9] M. N. Krein and S. V. Grek, On the spectrum properties of positive 1-degree maps, Modern Problems of Functional Analysis and Differential Equations. Proceedings of the Conference, Voronezh, 2003, pp. 161-163.

[10] M. N. Krein and T. N. Korobova, 1-degree maps in non-commutative algebras and Markov chains, Modern Methods of Function Theory and Adjoined Problems. Proceedings of Voronezh Winter Mathematical School, Voronezh, 2005, pp. 128-129.

[11] N. H. Kuiper, The homotopy type of the unitary group of Hilbert space, Topology 3 (1965), no. 1, 19-30.

[12] V. M. Manuilov and E. V. Troitsky, Hilbert C*-Modules, Factorial Press, Moscow, 2001.

[13] J. Milnor, On spaces having the homotopy type of CW-complex, Transactions of the American Mathematical Society 90 (1959), 272-280.

[14] A. S. Miščenko and A. T. Fomenko, The index of elliptic operators over $C^{*}$-algebras, Izvestiya Akademii Nauk SSSR. Seriya Matematicheskaya 43 (1979), no. 4, 831-859, 967 (Russian).

[15] V. E. Nazaikinskii, V. E. Shatalov, and B. Yu. Sternin, Methods of Noncommutative Analysis. Theory and Applications, de Gruyter Studies in Mathematics, vol. 22, Walter de Gruyter, Berlin, 1996.

[16] J. H. C. Whitehead, Combinatorial homotopy. I, Bulletin of the American Mathematical Society 55 (1949), 213-245.

Maria N. Krein: Faculty of Physics and Mathematics, Lipetsk State Pedagogical University, ul. Lenina 42, 398020 Lipetsk, Russia

E-mail address: travkin@lipetsk.ru 


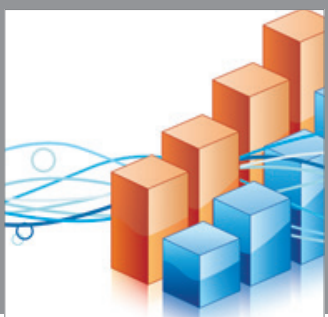

Advances in

Operations Research

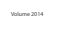

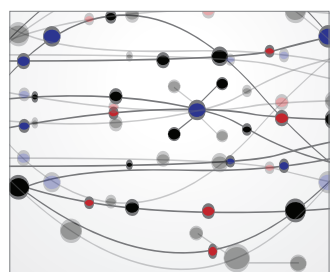

\section{The Scientific} World Journal
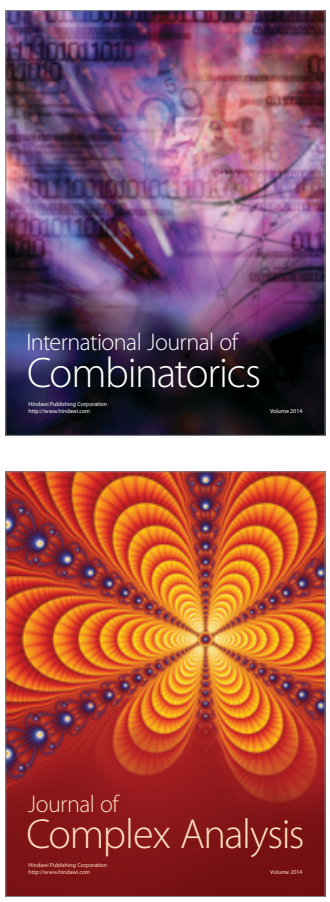

International Journal of

Mathematics and

Mathematical

Sciences
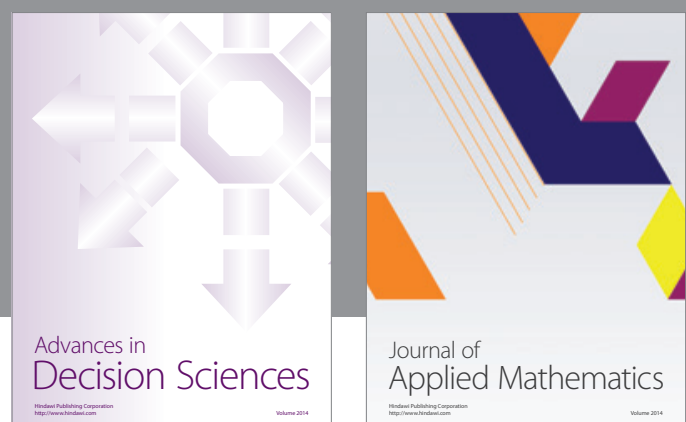

Journal of

Applied Mathematics
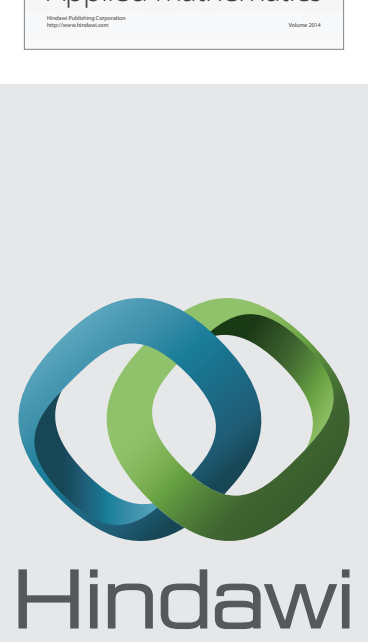

Submit your manuscripts at http://www.hindawi.com
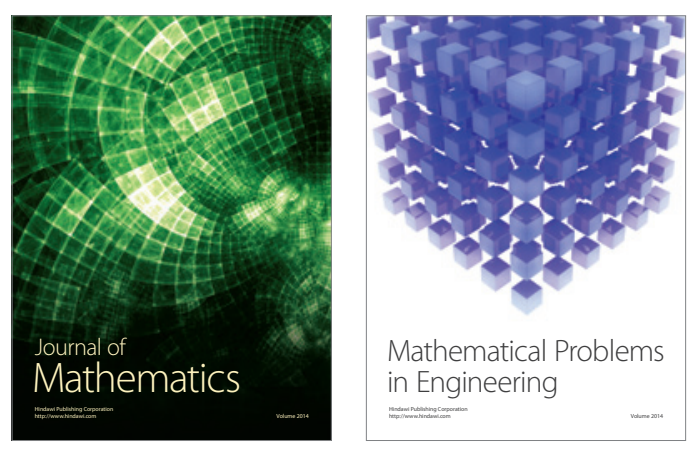

Mathematical Problems in Engineering
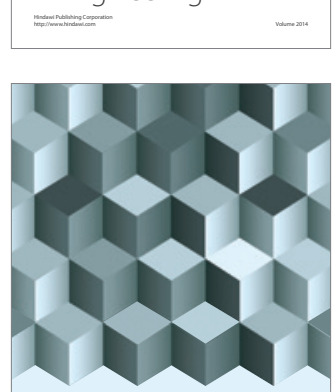

Journal of

Function Spaces
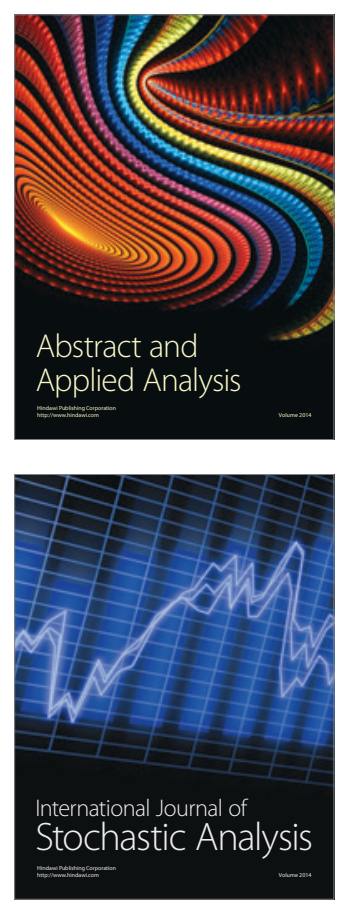

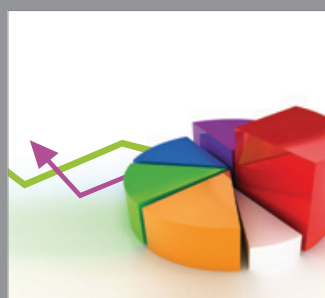

ournal of

Probability and Statistics

Promensencen
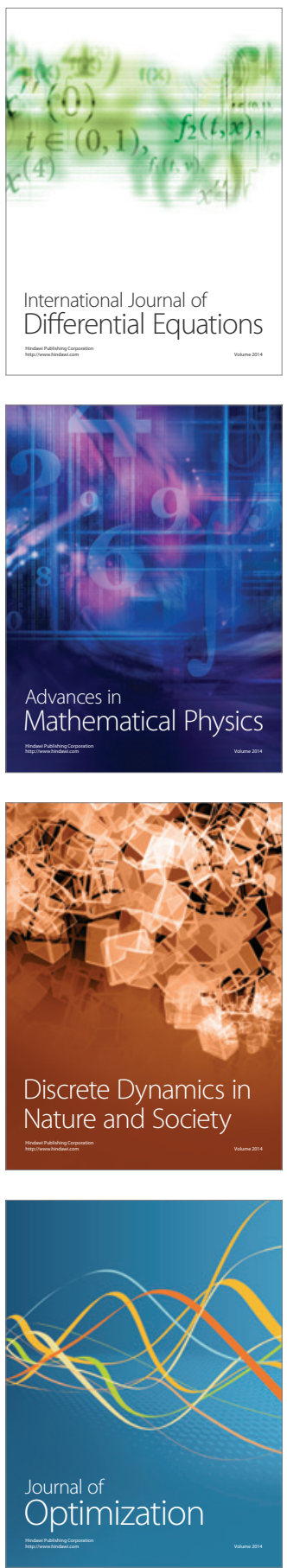